\title{
Stabilization Procedures for Near-Wall Full Reynolds Stress Closures in Complex 3-D CFD Simulation
}

$$
\text { CONF-9606418- }
$$

R. F. Kunz, B. W. Siebert, et. al.

June 1996

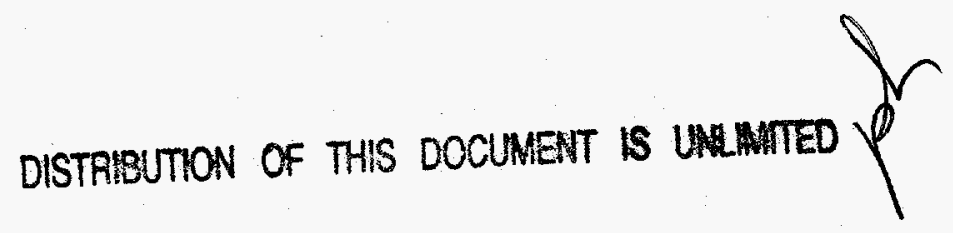

MASTER

\section{NOTICE}

This report was prepared as an account of work sponsored by the United States Government. Neither the United States, nor the United States Department of Energy, nor any of their employees, nor any of their contractors, subcontractors, or their employees, makes any warranty, express or implied, or assumes any legal liability or responsibility for the accuracy, completeness or usefulness of any information, apparatus, product or process disclosed, or represents that its use would not infringe privately owned rights. 


\section{DISCLAIMER}

This report was prepared as an account of work sponsored by an agency of the United States Government. Neither the United States Government nor any agency thereof, nor any of their employees, make any warranty, express or implied, or assumes any legal liability or responsibility for the accuracy, completeness, or usefulness of any information, apparatus, product, or process disclosed, or represents that its use would not infringe privately owned rights. Reference herein to any specific commercial product, process, or service by trade name, trademark, manufacturer, or otherwise does not necessarily constitute or imply its endorsement, recommendation, or favoring by the United States Government or any agency thereof. The views and opinions of authors expressed herein do not necessarily state or reflect those of the United States Government or any agency thereof. 


\section{DISCLAIMER}

Portions of this document may be illegible in electronic image products. Images are produced from the best available original document. 


\title{
Stabilization Procedures for Near-Wall Full Reynolds Stress Closures in Complex 3-D CFD Simulations
}

\author{
Robert F. Kunz, Brett W. Siebert \\ Lockheed Martin Corporation, Schenectady, NY 12301, 518-395-4495
}

\begin{abstract}
Near-wall full Reynolds stress models (FRSMs) were implemented in an implicit, incompressible full Navier-Stokes algorithm. The method was applied to flow in a 3-D duct with significant curvature induced secondary motions as well as 2-D and 3-D turbine cascade flows. FRSM computation of such flows, provides several numerical challenges germane to the very highly clustered near-wall meshes required, and to the incorporation of FRSMs themselves. Several stabilization procedures have been deployed within the scheme, which enable convergent solutions to large scale flow simulations of engineering interest. This paper summarizes the numerical strategies employed. It is shown that nearwall FRSM convergence rates commensurate with those of two-equation models are obtained.
\end{abstract}

\section{INTRODUCTION}

Near-wall turbulence models have made significant inroads into applied Computational Fluid Dynamics (CFD) environments in the past decade. A hierarchy has developed for these models, whose common feature is extended validity to regions where the local turbulence Reynolds number is low. Transport near-wall closures (two-equation and Reynolds stress) were introduced almost simultaneously with their more popular high Reynolds number forms in the mid-1970's, but remained impractical for 3-D flows until the mid-1980's. In the two-equation model class, the last decade has seen a clear transition away from high Reynolds number forms, engendered by vigorous and ongoing modelling research, and significant increases in digital computer capability. Numerous groups have also been actively pursuing near-wall FRS modelling since the first published model of this type was put forward in the 1970's.

In the near-wall FRSM class, significant inroads have not yet been made into applied CFD environments. There are a number of reasons for this including: • the lack of a body of literature which demonstrates successful deployment of FRSMs to complex 3-D flows, $\bullet$ the comparative immaturity of near-wall FRS turbulence modelling, • the general high level of complexity of near-wall FRSM formulations which may not be justified considering other simplifications/shortcomings germane to 3-D design codes, $\bullet$ increased computer resource requirements and analysis setup time compared to twoequation models and $\bullet$ numerical stability issues.

Indeed, published applications of near-wall FRS closures to any but very simple geometries are sparse. Few applications of near-wall FRSMs to 3-D configurations in full Navier-Stokes codes have appeared [Soritopoulos and Patel (1994), Aksoy (1995), for example]. What has motivated these authors, and the present work, is the good physical case which can be made for adopting a near-wall FRSM level of closure for many complex flows, despite the relative immaturity of these models. In particular, plausible arguments can be put forward for adopting this level of closure in configurations with "extra" strain rates due to large streamline curvature. Also, significant three-dimensionality can occur in near-wall boundary layers. Isotropic eddy viscosity approaches are wel-known to perform poorly in these extra strain rate and 3-D shear flows. Near-wall formulations mitigate the well documented shortcomings associated with wall functions. Specifically, the performance of such boundary treatments in flows with strong pressure gradients and in 3-D flows with strong secondary motion is poor.

In order that near-wall FRSMs reach the level of acceptance of near-wall two-equation models, the accuracy and usability issues indicated above must be adequately addressed. The focus of this work is on overcoming numerical issues associated with near-wall FRSMs in realistic engineering flows. A 3-D full Navier-Stokes algorithm is used as a platform for incorporation of near-wall two-equation and FRS closures. Two representative near-wall turbulence models are implemented, a two-equation model due to Chien (1982), and a FRSM model proposed by So and Yoo (1986). Several significant convergence issues which arise when applying the FRS systems to high Reynolds number 3-D flows are addressed. A new realizability enforcing source term treatment is introduced and is found to be more effective in stabilizing the scheme in early iteration than more conventional treatments.

The paper is organized as follows. First the theoretical formulation, including governing equations and numerical implementation is presented, with focus on the FRSMs. Results are then presented for a 3-D duct flow with significant curvature induced secondary motions and for 2-D and 3-D turbine cascade flow analyses. 


\section{THEORETICAL FORMULATION}

\section{Baseline Formulation}

A 3-D, pseudocompressibility based, implicit finite difference Navier-Stokes flow solver is used in the present investigations. The code solves the flow equations in generalized body fitted coordinates, using a diagonalized approximate factorization scheme. Inviscid fiux Jacobian eigenvalue based local timesteps and scalar artificial diffusion operators are employed. Several near-wall two-equation and

FRS models are installed. The governing equations, cast in generalized coordinates, can be written as:

$$
\begin{gathered}
\frac{\partial}{\partial t} \hat{\mathrm{Q}}+\frac{\partial}{\partial \xi}\left(\hat{E}_{1}-\hat{E}_{1 v}\right)+\frac{\partial}{\partial \eta}\left(\hat{E}_{2}-\hat{E}_{2 v}\right)+\frac{\partial}{\partial \zeta}\left(\hat{E}_{3}-\hat{E}_{3 v}\right)=S \\
\hat{Q}=J^{-1}\left(p / \rho, U_{1}, U_{2}, U_{3}, k, \varepsilon\right){ }^{T} \text { or } J^{-1}\left(p / \rho, U_{1}, U_{2}, U_{3}, \varepsilon, \overline{u_{1} u_{1}}, \overline{u_{2} u_{2}}, \overline{u_{3} u_{3}}, \overline{u_{1} u_{2}}, \overline{u_{2} u_{3}}, \overline{u_{3} u_{1}}\right)^{T}
\end{gathered}
$$

The vectors $\hat{E}_{i}, \hat{E}_{i v}$ are inviscid and viscous flux vectors. In turbulent flow, the form of the viscous fiux vectors depends on the model employed. Vector $\hat{s} \equiv \mathrm{S} / \mathrm{J}$ contains the turbulence model source terms. The particular form of the near-wall k- $\varepsilon$ and FRS models employed here are due to Chien (1982) and So and Yoo (1986). Briefiy, the So-Yoo model used here incorporates the Daly-Harlow turbulent diffusion model, the Launder-Reese-Rodi model 1 with near-wall corrections for pressure-redistribution, the Kolmogorov dissipation model, a near-wall production and rapid pressure correction function due to Hanjalic-Launder and Chien's transport dissipation equation.

For the mean flow, discrete equations are obtained by applying Euler implicit differencing, converting to $\Delta$-form, linearizing the LHS flux vectors, factoring the spatial operators in space, approximately diagonalizing the flux vectors, and introducing eigenvalue scaled implicit and explicit artificial dissipation operators. Second-order accurate central differences are used to discretize the inviscid and viscous fiuxes:

$$
\begin{gathered}
\left(T_{\xi}\left[I+J \Delta t \delta_{\xi}\left(\hat{\Lambda}_{i}-\hat{f}_{1}\right) \div S_{\xi} \delta_{2 \xi}\right] T_{\xi}^{-1}\right) \cdot\left(T_{\eta}\left[I+I \Delta t \delta_{\eta}\left(\hat{\Lambda}_{2}-\hat{H}_{2}\right)+S_{\eta_{i}} \delta_{2 \eta}\right] T_{\eta}^{-1}\right) \cdot\left(T_{5}\left[I+J \Delta t \delta_{\zeta}\left(\hat{\Lambda}_{5}-\hat{S}_{3}\right) \div S_{5} \delta_{2 \zeta}\right] T_{5}^{-1}\right) \Delta Q= \\
-J \Delta t\left[\delta_{\xi}\left(\hat{E}_{1}-E_{1 v}\right)+\delta_{\eta}\left(E_{2}-E_{2 v}\right)+\delta_{\zeta}\left(\hat{E}_{3}-E_{3 v}\right)+S_{\xi} \delta_{4 \xi} Q+S_{\eta} \delta_{4 \eta} Q+S_{\zeta} \delta_{4 \zeta} Q\right]^{\mathrm{n}}
\end{gathered}
$$

\section{Turbulence Model Implementation}

In the present work, turbulence transport equations are uncoupled from the mean flow equations. At each timestep, equation 2 is solved for the coupled mean fiow equations. Each of the discrete turbulence scalar equations are then solved in succession. Since these equations are uncoupled, this procedure requires the inversion of one scalar tridiagonal system in each direction for each equation:

$$
\begin{gathered}
{\left[1+j \Delta t \delta_{\xi}\left(\left(O_{1}-\hat{\gamma}_{1}\right)+S_{\xi} \delta_{2 \xi}\right)\right] \cdot\left[1+J \Delta t \delta_{\eta}\left(\left(O_{2}-\hat{\gamma}_{2}\right)+S_{\eta} \delta_{2 \eta}\right)\right] \cdot\left[1+J \Delta t \delta_{\zeta}\left(\left(O_{3}-\hat{\gamma}_{3}\right)+S_{\zeta} \delta_{2 \zeta}\right)\right] \Delta \phi=} \\
-J \Delta t\left[\delta_{\xi}\left(\hat{e}_{1}-\hat{e}_{1 v}\right)+\delta_{\eta}\left(\hat{e}_{2}-\hat{e}_{2 v}\right)+\delta_{\zeta}\left(\hat{e}_{3}-\hat{e}_{3 v}\right)+\hat{s}-S_{\xi} \delta_{2 \xi} \phi-S_{\eta} \delta_{2 \eta} \phi-S_{\zeta} \delta_{2 \zeta} \phi\right]^{n}
\end{gathered}
$$

\section{FRSM Stabilization Strategies}

The local timestep and artificial dissipation operators installed in the code are formulated to provide good convergence rates, and small but adequate smoothing on the very highly refined grids required for high Reynolds number computations using near-wall turbulence models. The necessity of these strategies arises primarily from anisotropy in grid spacing, independent of the turbulence model used. There are, however, additional stability issues which arise when FRSMs are employed, four of which are typically cited as contributing to these numerical difficulties:

1) Application of higher order convection numerics in the turbulence scalar equations. 2) Treatment of Reynolds stress terms in the momentum equations. 3) Treatment of source terms in the turbulence scalar equations. 4) Treatment of turbulence diffusion terms in the turbulence scalar equations (especially on non-orthogonal meshes). The methodologies employed in the present work to address these issues are summarized below.

The second difference explicit scalar artificial dissipation operator on the RHS of equation 3 corresponds closely to using first order upwind differencing for the convection terms. The stability afforded by such a treatment is one of the several reasons for the robustness of the present scheme for both two-equation and FRS near-wall models. Many researchers have adopted first-order upwinding for turbulence scalar convection, as the sensitivity of mean flow predictions to such a treatment is much smaller than if upwind or "hybrid" differencing schemes are used for the momentum equations.

The appearance of the term $\partial\left(-\overline{u_{i} u_{j}}\right) / \partial x_{j}$ in the momentum equations is generally not problematic when eddy viscosity models are used, since, by virtue of the Boussinesq approximation, this term is modelled as an effective diffusive transport. However, when Reynolds stress closures are employed, $\partial\left(-\overline{u_{i} u_{j}}\right) / \partial x_{j}$ must be discretized directly. This gives rise to two difficulties compared to eddy viscosity 
models. First, a straightforward implicit linearization of this term is not forthcoming, since the mean velocity component of the momentum equation under consideration does not appear. Second, central differencing applied to the stress divergence term can give rise to odd-even decoupling of the stresses on colocated grids. To address the first issue, Huang and Leschziner (1985) introduced a method for treating the Reynolds stress terms implicitly. In this approach, the discrete Reynolds stress equations are cast in a form where velocity gradient terms are collected whose coefficients are positive definite. These arise from components of the production and redistribution tensors. This approach has been employed successfully for a decade by several groups. Interestingly, this method relies on physical mechanisms which are categorically not diffusive in nature to provide apparent diffusion and, thereby, stability to the numerical method.

In this work, the absence of a naturally appearing turbulence diffusion term in the momentum equations is handled as follows. The Reynolds stress terms which appear in the momentum equations, $\partial\left(-\overline{u_{i} u_{j}}\right) / \partial x_{j}$, are explicitly added to the RHS of equation 2 , but the implicit accommodation of these terms is achieved by constructing an isotropic effective diffusion coefficient and installing this term as the entries in the diagonal viscous Jacobian:

$$
v_{t}=\frac{f_{\mu} C_{\mu}\left[\frac{1}{2} \bar{u}_{i} u_{i}\right]^{2}}{\varepsilon}, \quad \hat{\Gamma}_{i}=\frac{\nu_{t}}{J}\left[\operatorname{diag} 0 ;\left(\nabla \xi_{i} \cdot \nabla \xi_{i}\right) \frac{\partial}{\partial \xi_{i}},\left(\nabla \xi_{i} \bullet \nabla \xi_{i}\right) \frac{\partial}{\partial \xi_{i}},\left(\nabla \xi_{i} \bullet \nabla \xi_{i}\right) \frac{\partial}{\partial \xi_{i}}\right]
$$

In equation 4 , the form of $f_{\mu}$ is taken from Chien's $k-\varepsilon$ model (this form is computed anyway in the So-Yoo FRSM). This rather simple approach appears more robust in early iteration and on nonorthogonal meshes than the Huang-Leschziner approach or a related anisotropic effective diffusion construction treatment, and was used in all of the computations presented in this paper. This method introduces no additional approximations, since only the LHS is affected in the $\Delta$-formulation. Unlike the Huang-Leschziner approach, this method is not dependent on the form of the FRSM used, and exploits the predominantly diffusive nature of the Reynolds stress divergence in the momentum equations.

The checkerboarding which can occur, when central differences are applied to the stress divergence terms, is mitigated in staggered grid approaches where Reynolds stresses are stored at nodes staggered to the velocity component mesh. In colocated schemes, an apparently effective strategy, employed by several groups, is to couple the Reynolds stresses to the mean velocity in the same way as if a staggered variable arrangement were used. In the present colocated scheme, no such treatment is necessary. This is for the same reason that the pressure gradient treatment is not problematic in conventional time-marching procedures. Specifically, in such schemes, artificial diffusion which is either directly added (as here) or intrinsic to the upwinding employed, provide proper smoothing to all transport equations such that the $2 \Delta \mathrm{x}$ oscillations are minimized.

The careful treatment of source terms which appear in the Reynolds stress and dissipation rate equations also plays importantly in the stability of FRS methods. Specifically, the production, redistribution and dissipation terms which appear can give rise to unrealistically large values of the Reynolds stresses, especially in early iteration. Also problematic is the tendency of normal stresses and dissipation rate to become negative, especially in early iteration and in coarser grid regions of the flow field domain. It has been widely experienced that this has a destabilizing effect on iterative procedures for two-equation and FRS models. The authors have investigated two source treatment approaches to maintain positivity of normal stresses and dissipation. The first is similar to the simple proposal of Huang and Leschziner (1985). The normal stress and dissipation rate equations can be written symbolically as:

$$
\frac{d \phi}{d t}=\text { other terms }+s_{\mathrm{p}}^{+}+s_{\mathrm{p}}^{-}+s_{\Phi}^{+}+s_{\Phi}^{-}, s_{\Phi}^{+}=\operatorname{MAX}\left(s_{\Phi}, 0\right), s_{\Phi}^{-}=\operatorname{MIN}\left(s_{\Phi}, 0\right) \text {. }
$$

Where, $\mathrm{s}^{+/-}$represent components of the production and redistribution terms. Defining $s^{+}=s_{\mathrm{p}}^{+}+s_{\Phi^{*}}^{+}, s^{-}=s_{\mathrm{p}}^{-}+s_{\Phi}^{-}$, these source terms are implemented in each operator of equation 2 as:

$$
\left[1+\mathrm{J} \Delta t \delta_{\mathrm{Fi}}\left(\left(\hat{O}_{\mathrm{i}}-\hat{\gamma}_{\mathrm{i}}\right)+\mathrm{S}_{\mathrm{i}} \delta_{2 \mathrm{i}}-\frac{\mathrm{f}}{\frac{\mathrm{f}}{3}} \frac{\hat{\mathrm{s}}_{\mathrm{q}}^{\mathrm{n}}}{\mathrm{n}}\right], 0 \leq \mathrm{f} \leq 1\right.
$$

This treatment can maintain positivity of positive quantities (indeed it can be shown that it must for diagonal dominant discretizations if all potentially negative terms are included in $\hat{s}^{-}$). However, in early iteration this treatment can fail to maintain positivity, and importantly, it can lead to slow convergence rates due to the potentially excessive diagonal dominance it can provide. This "relaxation" effect arises due to the treatment of only destruction terms implicitly. 
a
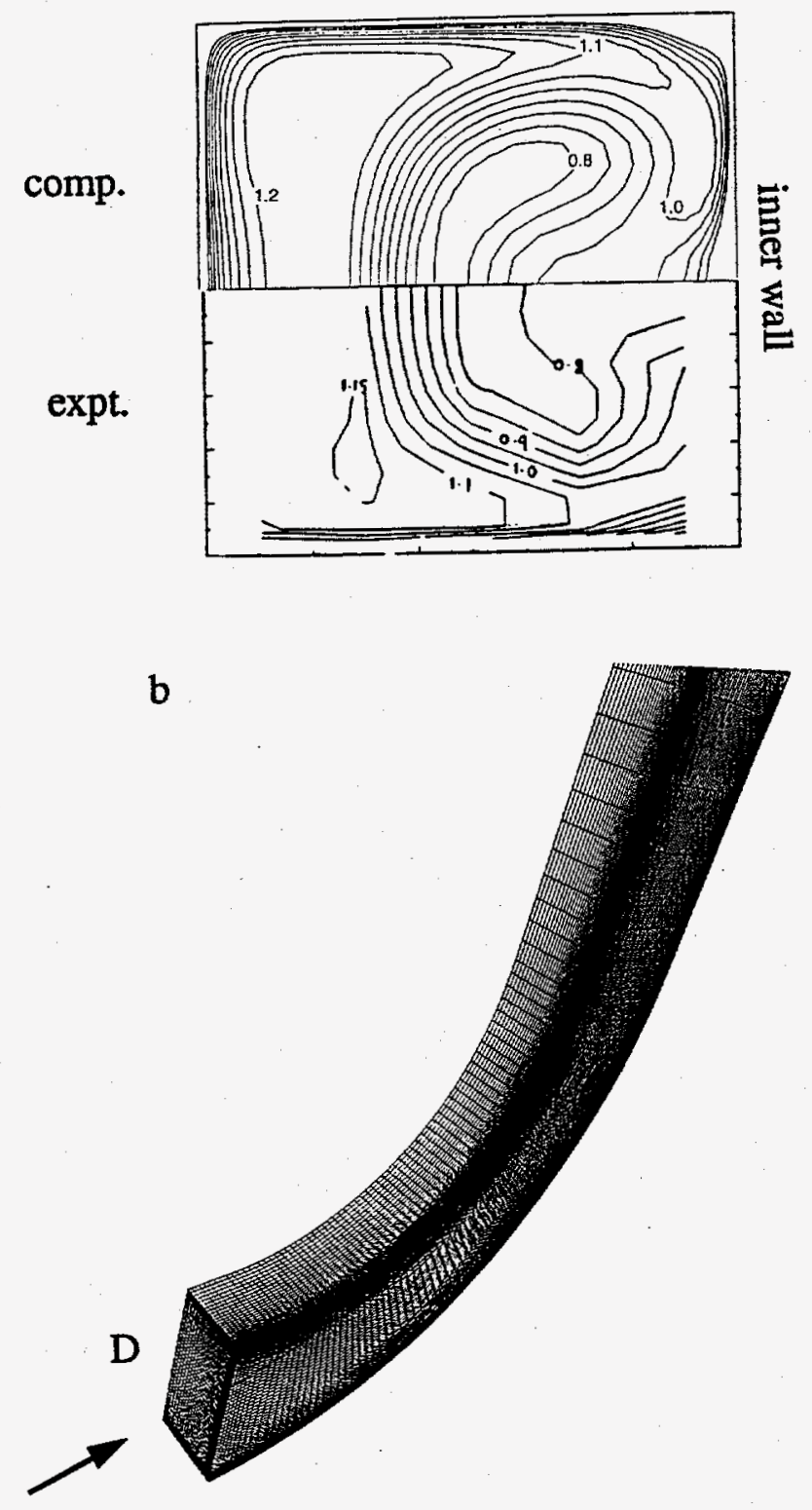

symmetry plane

c
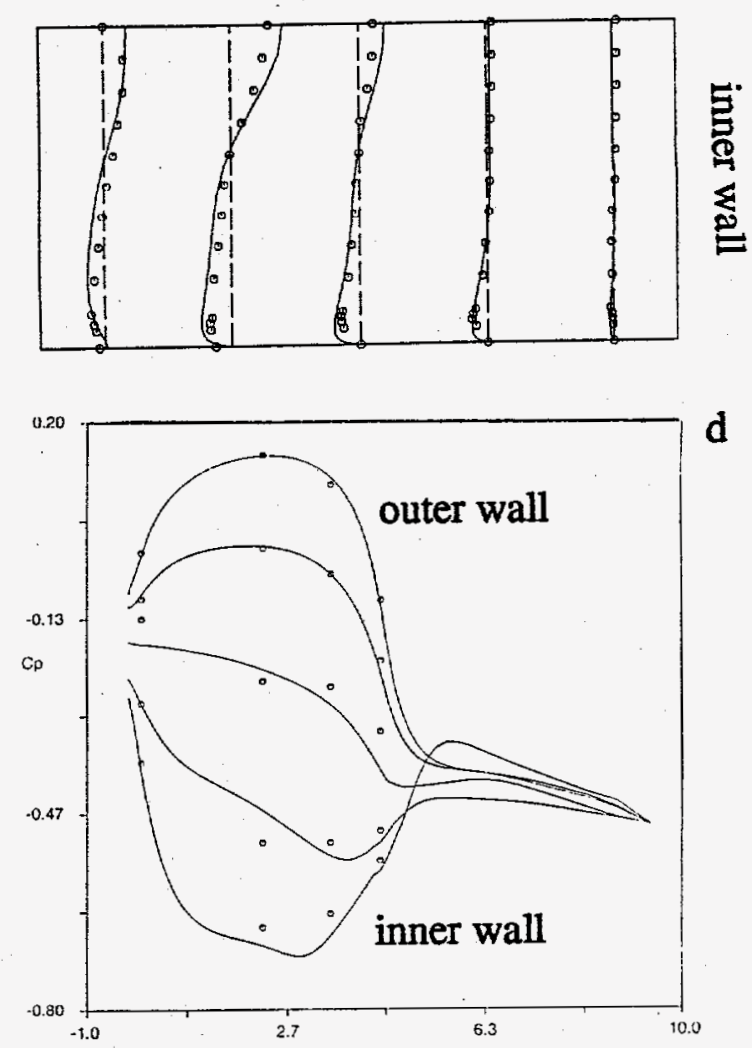

e



Figure 1. Taylor Duct Results. a) Predicted vs. Measured Streamwise Velocity Contours 2.5 Hydraulic Diameters Downstream of Bend Exit. b) View of $81 \times 51 \times 101$ grid. c) Predicted vs. Measured Secondary Flow Velocities 2.5 Hydraulic Diameters Downstream of Bend Exit.

d) Predicted vs. Measured Static Pressure Coefficients at 5 Radial Locations (z/D = .1, .3, .5, .7, .9) On Duct Symmetry Plane. e) Predicted Normal Stress Profiles along Symmetry Plane at Bend Exit.

A new proposal is employed here which mitigates these issues. It's development arises from a method used in multi-phase flow calculations to prevent volume fractions from becoming too small $(<0)$ and too large $(>1)$ [Siebert et al. (1995)]. The method is analogous to under-relaxation, except the anchor value is not the value from the previous iteration (or timestep), it is the limiting value which the variable can achieve. In FRSM applications, all turbulence scalars are prevented from becoming too large or too small during a given iteration. The limits can be physical, as in a lower limit of zero for the normal stresses and dissipation, or they can be based on a bound of the turbulent kinetic energy or on a maximum change from the current value. Regardless, the method has no impact on the converged results. The treatment develops from the idea that a source tends to increase the variable, so to prevent the variable from exceeding a given value, the source term must be less than some limiting value. A similar condition holds for a sink. The resulting scheme 

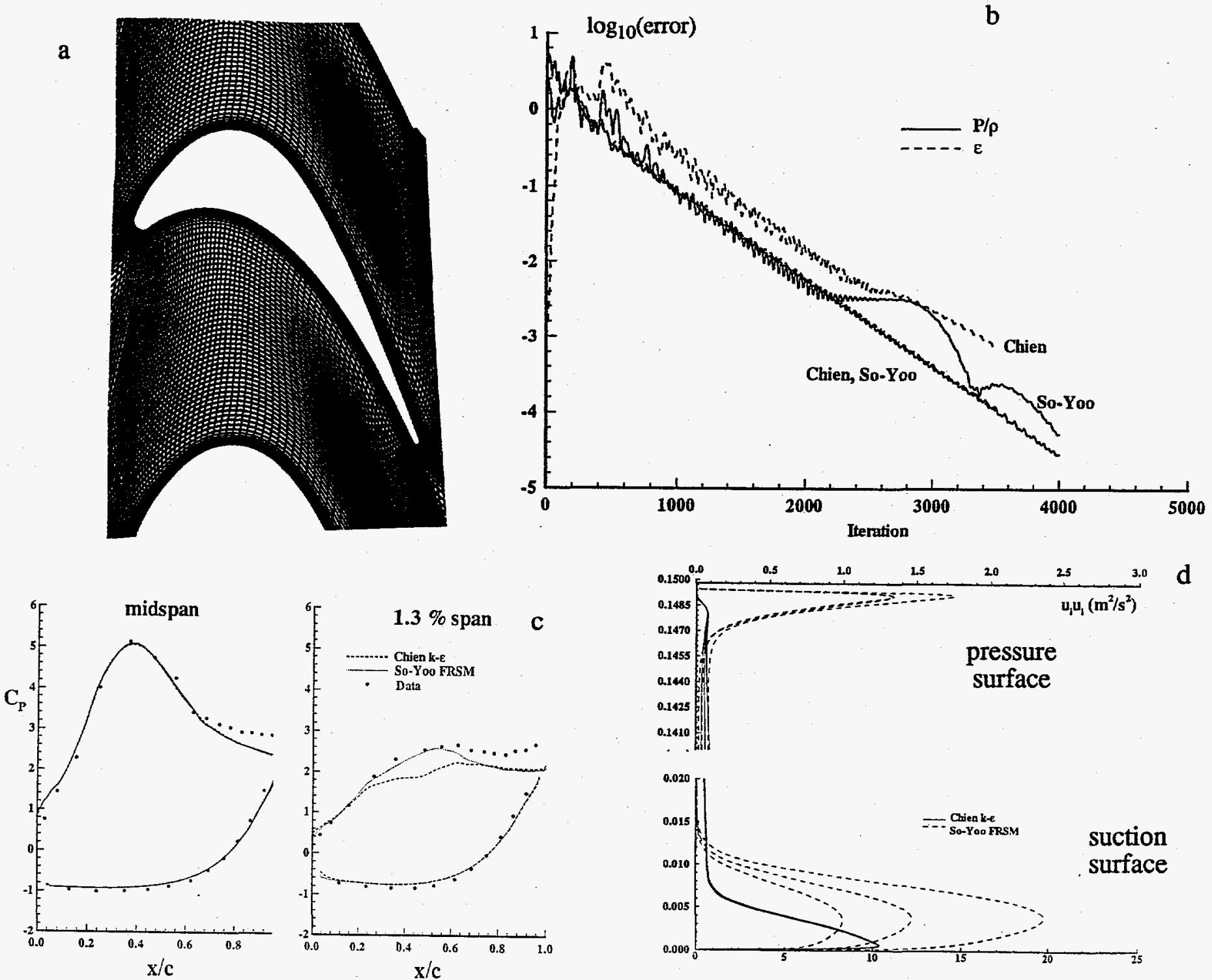

d
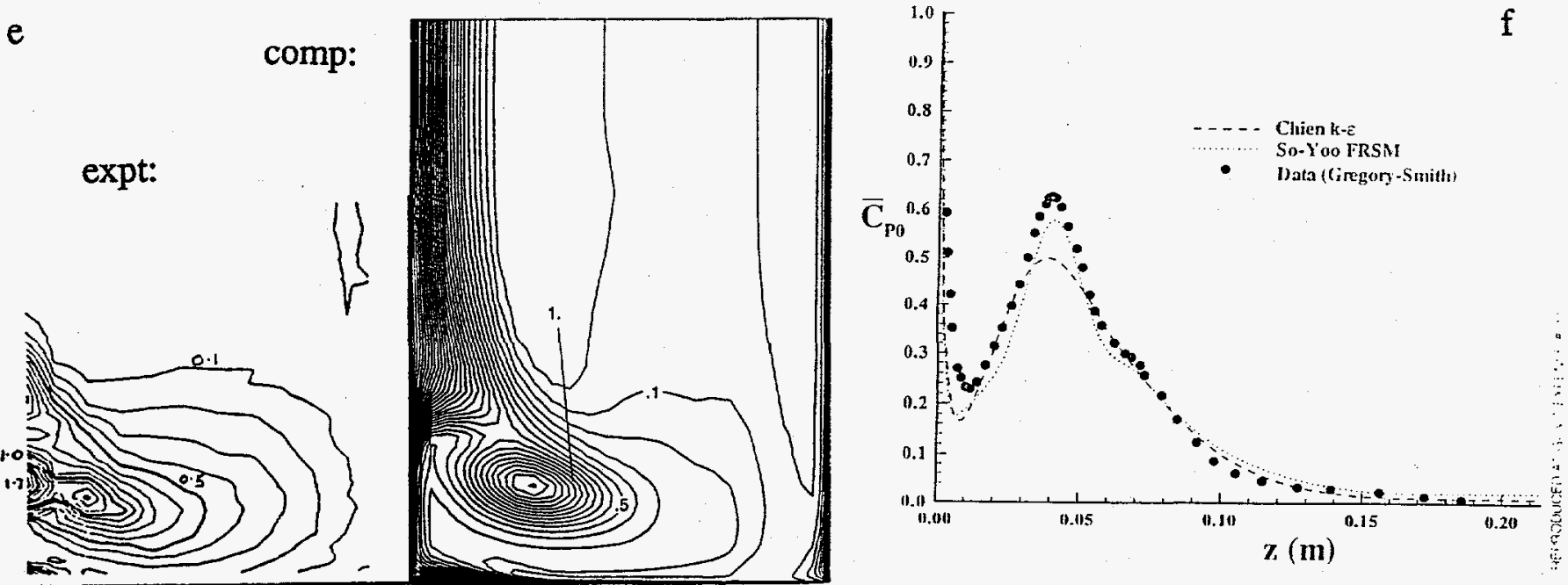

Figure 2. Gregory-Smith Turbine Cascade Results. a) View of $125 \times 185$ Grid Used for 2D Simulations. b) Comparison of k- $\varepsilon$ and FRSM Convergence Histories for 2D Simulations. c) Predicted vs. Measured Blade Pressure Distributions. d) Predicted Normal Stress Profiles at 71\% Chord. e) Predicted vs. Measured Stagnation Pressure Loss Coefficient at $87 \%$ Chord.

f) Predicted vs. Measured Mass-Weighted Spanwise Distribution of Loss Coefficient at $87 \%$ Chord. 
is described here for a diagonal dominant discretization.

$$
\begin{aligned}
& A_{p}^{i j} \overline{u_{i} u_{j}}=\sum A_{k}^{i j} \overline{u_{i} u_{j}}+S^{i j}+\max \left(R_{\min }^{i j}, \min \left(R_{\max }^{i j}, R^{i j}\right)\right) \\
& R_{\max (\min )}^{\mathrm{ij}}=A_{\mathrm{p}}^{\mathrm{ij}}\left(\overline{\mathrm{u}_{\mathrm{i}} \mathrm{u}_{\mathrm{j}}}\right)_{\max (\min )}-\left(\sum \mathrm{A}_{\mathrm{k}}^{\mathrm{A}_{\mathrm{u}}^{\mathrm{ij}} \overline{\mathrm{u}}_{\mathrm{j}}}+\mathrm{S}^{\mathrm{ij}}\right) \\
& \mathrm{r}_{+}^{\mathrm{ij}}=\max \left(\mathrm{R}^{\mathrm{ij}}, 0\right) \quad ; \quad \mathrm{r}_{-}^{\mathrm{ij}}=\min \left(\mathrm{R}^{\mathrm{ij}}, 0\right)
\end{aligned}
$$

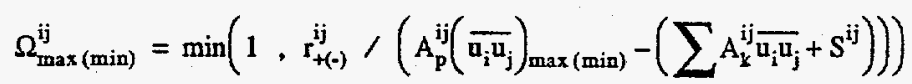

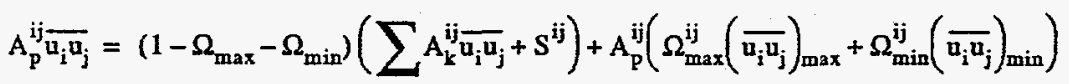

If the source that is to be introduced into the linear equations, $\mathrm{R}^{\mathrm{ij}}$, is larger that the value, $\mathrm{r}^{\mathrm{ij}}$, (determined explicitly) that would result in the limiting variable, then the $\Omega$ factor becomes one, and the equations return the limiting variable. Otherwise, the source term, $R^{13}$, is introduced not as an explicit constant, but as a percentage of the limit, where the limit changes with the updates of the neighboring variables. Specifying a minimum/maximum variable simultaneously sets a minimum/maximum change in the variable. This scheme is implemented in a factored implicit scheme is a straightforward manner.

The last important stability issue related to FRSMs is the treatment of the turbulent diffusion terms in the stress and dissipation rate equations themselves. The three most commonly employed models for this term are those due to $\bullet$ Shir, $\bullet$ Daly and Harlow and $\bullet$ Hanjalic and Launder. In 3-D applications, on body-fitted grids, the choice of the turbulent diffusion model has a significant impact on the numerical stability and CPU performance of the overall scheme. In 3-D generalized coordinates, Shir's model gives rise to 27 terms of the form:

$$
\frac{\partial}{\partial \xi}\left(\xi_{x}\left(\frac{C_{3} k}{j \varepsilon}\right) \overline{u_{1} u_{1}} \xi_{x} \frac{\partial}{\partial \xi}\left(\overline{u_{1} u_{1}}\right)\right) .
$$

Nine of these terms can be treated implicitly. The six off-diagonal terms in equation 12 are zero on an orthogonal grid. Accordingly, this model is quite stable on near-orthogonal meshes. Because of its relative simplicity, the Shir model does not burden the overall CPU performance of the method. In generalized coordinates, the Daly-Harlow model expands to 81 terms of the form of equation 12, nine of which can be treated implicitly. It has been the experience of these authors and others that the numerous cross diffusion terms which appear are numerically destabilizing, especially in 3-D. However, by inclusion of negative terms which arise in this expansion, as part of the source treatment detailed above, this model is observed to be nearly as numerical stable as Shir's model in the present scheme. Importantly, however, is the relative computational intensity of implementing the Daly-Harlow model. Three-dimensional run times of the code are increased by over $30 \%$ per timestep merely by adopting the Daly-Harlow model over the Shir model.

Several researchers have recommended and used the Hanjalic-Launder model, arguing for its tensorial consistency with the exact terms and somewhat improved predictive capabilities in, for example, rearward facing step flow. In 3-D body fitted applications, 243 terms of the form of equation 12 appear. It has not been demonstrated in the literature that improved accuracy can be achieved using the Hanjalic-Launder model in complex 3-D flows, though the full implementation of this model required in such flows would severely burden the stability and CPU performance of the scheme.

\section{RESULTS}

\section{3-D Flow in a $90^{\circ}$ Bending Duct of Square Cross-Section}

The first test case presented is an incompressible turbulent duct flow tested by Taylor et al. (1981). The geometry of the duct is shown in Figure $1 \mathrm{~b}$. The Reynolds number based on hydraulic diameter and bulk velocity was $4.0 \times 10^{4}$. The curvature ratio of the duct, $\mathrm{r}_{\mathrm{i}} / \mathrm{D}_{\mathrm{H}}=1.8$. LDV data of streamwise and radial velocities and static pressure probe measurements are avallable. The $81 \times 51 \times 101$ grid used for the simulation is shown in Figure 1- the symmetry of the configuration was exploited. A four order of magnitude decrease in RMS residuals of all equations was achieved in 1500 iterations. Incorporation of the source term treatments specified above allowed the simulation to be initialized from flat mean velocity and turbulence quantity profiles with the same CFL used for both turbulence model and mean flow equations. Without this treatment, the CFL $\mathrm{RS}_{\mathrm{RS}}$ had to be reduced in early iteration to $0.1 \mathrm{CFL}_{\text {mean }}$, and the solution could be restarted with $\mathrm{CFL}_{\mathrm{RS}}=1.0$ after 1000 iterations. 
Figure 1a shows predicted and measured streamwise velocity contours 2.5 hydraulic diameters downstream of the bend exit. The analysis does an excellent job in predicting the streamwise flow development which is characterized at this location by a "pinching off" of low momentum fluid entrained from the wall shear layers by secondary flow. Figure 1c shows a direct comparison of predicted and measured secondary flow at this streamwise station. Again, the prediction of these crossflow secondary motions are well captured by the FRS analysis. The static pressure field which drives the secondary flow is well predicted as shown in Figure 1d, which compares the FRS solution to streamwise distributions of pressure coefficient at several radial locations on the duct symmetry plane. Figure le shows predicted profiles of streamwise and radial normal Reynolds stresses on the symmetry plane at the bend exit. Results obtained using the $k-\varepsilon$ model are provided for comparison. The FRS model exhibits significant stress anisotropy especially on the inner wall, and also exhibits an amplification of turbulence intensity compared to the two-equation model on the convex outer wall surface.

\section{2-D and 3-D Flow in a Linear Turbine Rotor Cascade}

The second test case presented is a high Reynolds number 3-D turbine rotor cascade tested by Gregory-Smith et al. (1988). The flow field in such cascades is characterized by significant secondary thow in the endwall region. Specifically, the incoming endwall boundary layer impinging upon the leading edge separates into a horseshoe vortex. The pressure side leg of this vortex merges with secondary flow induced by the turning of the endwall boundary layer, collectively forming the passage vortex which impinges upon the suction side of the adjacent blade.

A top view of the cascade is shown in Figure $2 \mathrm{a}$. The span/pitch ratio of the cascade is 2.4. The Reynolds number based on axial chord and inlet velocity was $5.0 \times 10^{5}$. Hot wire and five-hole pressure probe data provided mean velocities, selected turbulence statistics and static pressure. Both 2-D and 3-D simulations were performed. The $125 \times 185$ grid used for the 2-D simulations is shown in Figure $2 \mathrm{a}$. Grid studies showed that predicted blade skin friction varied by no more $3 \%$ along the blade with this grid compared to a $125 \times 93$ "halved" grid solution. Based on this, a $125 \times 93 \times 85$ mesh was utilized for the 3-D simulations. One half of the linear cascade was computed based on symmetry. Figure $2 \mathrm{~b}$ shows a comparison of FRS and $\mathrm{k}-\varepsilon$ convergence histories for the fine 2-D grid. The models exhibit similar convergence behaviour, achieving a 5 order of magnitude drop in residual RMS in 4000 iterations. The convergence rate for this case is slower than the Taylor duct due to the high grid stretching (and concomitant lower local timestep) required at the higher Reynolds number. The 3-D solutions converged to a 3.5 order of magnitude drop in residual RMS in 2000 iterations.

Blade static pressures are displayed in Figure $2 \mathrm{c}$. The FRS and $\mathrm{k}-\varepsilon$ models perform almost identically in the prediction of this quantity at midspan. At $1.3 \%$ span, differences appear. The data exhibits an unloading of the suction surface and a migration of the suction peak to approximately $60 \%$ chord (vs. $\cong 40 \%$ chord at midspan). This arises due to the impingement of the passage vortex on the suction surface. The FRSM model more closely matches the measured distribution to about $60 \%$ chord.

Figure 2d shows a comparison of near-wall Reynolds normal stress profiles (transformed to local grid aligned coordinates) at $71 \%$ chord for the $k-\varepsilon$ and FRS models. On the pressure surface the $k-\varepsilon$ model displays a typical near-wall turbulent kinetic energy profile, but as expected predicts almost no stress anisotropy. The So-Yoo model predicts a significantly more energetic and thicker boundary layer at this location, as well as a large degree of anisotropy. On the pressure surface, the $\mathrm{k}-\varepsilon$ model predicts an essentially laminar boundary layer. The So-Yoo model predicts low energy but clearly turbulent and anisotropic normal stress profiles there.

Figure 2e shows a comparison of experimentally measured total pressure loss coefficient at $87 \%$ chord with predictions using the FRSM. The location and strength of the loss core are well predicted. Predicted losses at this location are cast in mass-weighted pitch averaged form in Figure $2 f$. The $k-\varepsilon$ model provides a more accurate loss prediction near the endwall. The FRSM appears to yield superior peak core loss predictions.

\section{REFERENCES}

Aksoy, H. (1995) "Effects of Numerical Techniques and Near-Wall Modelling on the Prediction of Complex Turbulent Flows," Ph.D. Thesis, Mechanical and Aerospace Engineering, Arizona State University.

Chien, K. (1982) "Predictions of Channel and Boundary Layer Flows with a Low-Reynolds Number Turbulence Model," AlAA Journal, 20: 33.

Gregory-Smith, D. G., Walsh, J. A., Graves, C. P., Fulton, K. P. (1988) “Turbulence Measurements 
and Secondary Flows in a Turbine Rotor Cascade," ASME Joumal of Turbomachinery 110: 479.

Huang, P. G., Leschziner, M. A. (1985) "Stabilization of Recirculating-Flow Computations Performed With Second-Moment Closures and Third-Order Discretizations," Proceedings of 5th Symposium on Turbulent Shear Flow, 20.7.

Siebert, B. W., Maneri C. C., Kunz R. F., Edwards D. P. (1995)“A Four-Field Model and CFD Implementation for Multi-Dimensional, Heated Two-Phase Flows," Proc. 2nd Int. Conf. on Multiphase Flow, Kyoto, Japan, pp. P2:19-27.

So, R. M. C., Yoo, G. J. (1986) "On the Modeling of Low-Reynolds-Number Turbulence," NASACR-3994.

Soritopoulos, F., Patel, V. C. (1994) "Prediction of Turbulent Flow Through a Transition Duct Using a Second Moment Closure," AIAA Journal, 32: 2194.

Taylor, A.M.K.P., Whitelaw, J.H.,Yianneskis, M. (1981) "Measurements of Laminar and Turbulent Flow in Curved Duct With Thin Inlet Boundary Layers," NASA CP-3367. 